\title{
What is the role of neoadjuvant chemotherapy, radiation, and adjuvant treatment in resectable esophageal cancer?
}

\author{
Nasser Altorki, Sebron Harrison \\ Department of Cardiothoracic Surgery, Division of Thoracic Surgery, New York Presbyterian Hospital, Weill Cornell Medical College, New York, \\ NY, USA \\ Correspondence to: Dr. Sebron Harrison, MD. Division of Thoracic Surgery, Department of Cardiothoracic Surgery, Ste M404, Weill Cornell \\ Medicine, 525 E 68th St, New York, NY 10065, USA. Email: swh9002@med.cornell.edu.
}

\begin{abstract}
The majority of patients with operable esophageal cancers present with locally advanced disease, for which surgical resection as a sole treatment modality has been historically associated with poor survival. Even following radical resection, most of these patients will eventually succumb to their disease due to distant metastasis. For this reason, there has been intense interest in the role of neoadjuvant therapy. Neoadjuvant therapy primarily consists of either chemotherapy, radiation therapy, or a combination of the two. Multiple studies of variable scope, design, and patient characteristics have been conducted to determine whether neoadjuvant therapy is warranted, and-if so-what is the best modality of treatment. Despite nearly three decades of study, decisions regarding neoadjuvant therapy for esophageal cancer remain controversial. Regardless, the available evidence provided by large, prospective studies supports preoperative chemotherapy as opposed to surgery alone. Therefore, in our opinion, there is no longer any question as to whether induction therapy is appropriate for locally advanced esophageal cancer. Less clear, however, is the evidence that the addition of radiation to chemotherapy in the preoperative setting is superior to neoadjuvant chemotherapy alone. Our group generally advocates for neoadjuvant chemotherapy alone followed by radical esophageal resection. The data for adjuvant therapy are soft, and particularly troubling is the high rate of treatment drop out in trials studying adjuvant therapy. Therefore, we strongly prefer neoadjuvant chemotherapy and reserve adjuvant chemotherapy for those rare, highly selected patients - patients with T1 tumors, for example - who do not receive neoadjuvant treatment and are found to have occult nodal disease at the time of surgery.
\end{abstract}

Keywords: Esophageal cancer; neoadjuvant chemotherapy; radiation

Submitted Dec 06, 2016. Accepted for publication Mar 13, 2017.

doi: 10.21037/acs.2017.03.16

View this article at: http://dx.doi.org/10.21037/acs.2017.03.16

The majority of patients with operable esophageal cancers present with locally advanced disease, for which surgical resection as a sole treatment modality has been historically associated with poor survival. Local and systemic recurrences develop in over $80 \%$ of surgically treated patients, typically within 6-12 months postoperatively, and are often rapidly fatal. This pattern of local and systemic failure highlights the shortcomings of surgical resection alone as a modality of local tumor control, as well as the high propensity of the disease for early occult systemic dissemination. These observations have driven interest in multimodality treatment approaches dating back to the early 1980s with the objective of improving survival by enhancing loco-regional disease control and eliminating micrometastatic disease. Three major induction modalities have been employed, including preoperative chemotherapy, preoperative radiotherapy, or a combination of the two. Despite the large number of randomized trials conducted to date, controversy persists regarding the treatment of choice for patients with locally advanced esophageal cancer. In this communication, we discuss the pertinent level 1 evidence as it relates to the following questions: is 


\begin{tabular}{|c|c|c|c|c|c|c|c|c|c|c|c|}
\hline Reference & Trial & $\begin{array}{l}\text { Treatment } \\
\text { CS only }\end{array}$ & Treatment cohort & CS & $S$ & CS & S & CS & $S$ & CS & $S$ \\
\hline $\begin{array}{l}\text { Kelsen DP, et al. } \\
\text { (2007) (1) }\end{array}$ & $\begin{array}{l}\text { North American } \\
\text { (USA Intergroup } \\
113 \text { ) }\end{array}$ & $\begin{array}{l}\text { Cisplatin } \\
+\mathrm{FU}\end{array}$ & $\begin{array}{l}\text { Localized esophageal cancer } \\
\text { (50/50 adenocarcinoma/ } \\
\text { epidermoid) }\end{array}$ & 216 & 227 & 20 & 20 & - & - & 63 & 59 \\
\hline $\begin{array}{l}\text { Allum WH, et al. } \\
\text { (2009) (2) }\end{array}$ & $\begin{array}{l}\text { European } \\
\text { (OEO2) }\end{array}$ & $\begin{array}{l}\text { Cisplatin } \\
+\mathrm{FU}\end{array}$ & $\begin{array}{l}\text { Localized esophageal } \\
\text { cancer (>60\% each arm) }\end{array}$ & 400 & 402 & 23 & 17.1 & - & - & - & - \\
\hline $\begin{array}{l}\text { Cunningham D, } \\
\text { et al. (2006) (3) }\end{array}$ & MAGIC & ECF & $\begin{array}{l}\text { Adenocarcinoma of lower } \\
\text { esophagus, GEJ, or stomach }\end{array}$ & 250 & 253 & 36.3 & 23 & - & - & 69.3 & 66.4 \\
\hline
\end{tabular}

neoadjuvant treatment warranted? If so, what is the optimal neoadjuvant regimen? What is the role of surgery in the setting of modern chemotherapy and radiation therapy, where definitive chemoradiotherapy is increasingly utilized? Is there a role for adjuvant therapy? With these questions in mind, we have reviewed what we consider to be some of the most important recent studies highlighting the management of esophageal cancer- particularly in the neoadjuvant setting-and offer our perspective on managing such a complex and deadly disease.

\section{Is neoadjuvant therapy warranted?}

\section{Neoadjuvant chemotherapy}

At least 14 randomized trials (1-6) have compared preoperative chemotherapy followed by surgery (CS) with surgery alone (S). The majority of these trials are vastly statistically underpowered to permit meaningful conclusions from their results. The four appropriately powered trials that are frequently cited in the esophageal literature are summarized in Table 1. Two of these trials, reported around the turn of the millennium, compared perioperative cisplatin (Cis) and fluorouracil (FU) followed by surgery with surgery alone. The North American trial (USA Intergroup 113) (1) randomized 443 patients ( $50 \%$ adenocarcinoma). The rate of R0 resection was essentially equivalent between the two arms (CS: 63\%; S: 59\%), and the overall 5-year survival was identical at 5 and 7 years (20\%). The larger European trial (OEO2) (2) randomized 400 patients to CS and 402 patients to $\mathrm{S}$ only. The rate of adenocarcinoma was over $66 \%$ in both arms. Both R0 resection rates and number of nodal metastases favored the CS arm (CS: $60 \%$ vs. S: $54 \%$ and CS: $48 \%$ vs. S: $58 \%$, respectively). Overall survival at 5 years also favored the CS arm (CS: $23 \%$ vs. S: $17 \%$ ) and was consistent for both squamous cell carcinoma and adenocarcinoma. Unfortunately, these conflicting results did not bring the desired clarity to the utility of chemotherapy in the preoperative setting.

In the ensuing decade two additional trials investigated the role of preoperative chemotherapy specifically in patients with adenocarcinoma of the esophagus and gastroesophageal junction (GEJ). The larger of these trials by Cunningham, et al. (MAGIC Trial) (3) randomized 503 patients with carcinoma of the stomach, GEJ, and distal esophagus to either 3 preoperative cycles of epirubicin, cisplatin, and fluorouracil (ECF) followed by surgery and 3 additional cycles of ECF or to surgery alone. Patients with cancers of the GEJ and lower esophagus accounted for roughly $25 \%$ of patients in each arm. Overall 5 -year survival was $36.3 \%$ in the perioperative chemotherapy arm and $23 \%$ in the surgery alone arm without evidence of heterogeneity of treatment effect based on tumor location.

Based on the size and rigorous design of the MAGIC trial, preoperative chemotherapy with ECF is now considered one standard of care for the treatment of 


\begin{tabular}{|c|c|c|c|c|c|c|c|c|c|c|c|}
\hline \multirow[t]{2}{*}{ Reference } & \multirow[t]{2}{*}{ Trial } & \multirow{2}{*}{$\begin{array}{l}\text { Treatment } \\
\text { CRS }\end{array}$} & \multirow[t]{2}{*}{ Treatment cohort } & \multicolumn{2}{|c|}{$\begin{array}{l}\text { Patients } \\
\text { randomized } \\
\text { (no.) }\end{array}$} & \multicolumn{2}{|c|}{$\begin{array}{l}\text { OS 5-yr } \\
\text { rate (\%) }\end{array}$} & \multicolumn{2}{|c|}{$\begin{array}{l}\text { Disease- } \\
\text { free } 5-y r \\
\text { rate }(\%)\end{array}$} & \multicolumn{2}{|c|}{$\begin{array}{l}\text { Ro } \\
\text { resection } \\
(\%)\end{array}$} \\
\hline & & & & CRS & $S$ & CRS & $S$ & CRS & $S$ & CRS & $S$ \\
\hline $\begin{array}{l}\text { van Hagen P, } \\
\text { et al. (2012) (7) }\end{array}$ & $\begin{array}{l}\text { Dutch Cancer } \\
\text { Foundation } \\
\text { CROSS Trial }\end{array}$ & $\begin{array}{l}\text { Carboplatin } \\
+ \text { Paclitaxel, } \\
\text { concurrent } \\
\text { radiation }\end{array}$ & $\begin{array}{l}\text { Esophageal or GEJ cancer or } \\
\text { squamous }(23 \%) \text {, adenocarcinoma } \\
(75 \%) \text {, other }(2 \%) \text { origin. }\end{array}$ & 178 & 188 & 47 & 34 & - & - & 92 & 69 \\
\hline
\end{tabular}

operable adenocarcinoma of the esophagus and GEJ in Europe and to some extent in the United States.

Further support for preoperative chemotherapy for esophageal adenocarcinoma comes from a phase III trial conducted in France by the Fédération Nationale dex Centres de Lutte contre le Cancer (FNCLCC) and the Fédération Francophone de Cancérologie Digestive (FFCD), comparing perioperative chemotherapy (Cis $+\mathrm{FU}$ ) and surgery to surgery alone (4). In contrast to MAGIC, the French trial had a much higher percentage of patients with lower esophageal or GEJ adenocarcinoma ( $75 \%$ vs. $26 \%$ ). The investigators reported a significant improvement in 5 -year overall survival in the chemotherapy and surgery group compared to surgery alone $(38 \% v s$. $24 \%$, respectively). There were some obvious limitations to these large perioperative chemotherapy trials, including the lack of modern staging modalities such as endoscopic ultrasound /positron emission tomography. Additionally, fewer than $50 \%$ of eligible patients received their assigned post-operative chemotherapy. Nevertheless, the available evidence supports the superiority of preoperative chemotherapy over surgery alone as an acceptable multimodality standard of care for patients with adenocarcinoma of the esophagus and GEJ.

\section{Neoadjuvant chemoradiation}

At least 13 randomized trials (7-13) have investigated the role of preoperative chemoradiation (CRS) versus surgery alone (S). Seven trials investigated CRS exclusively in patients with squamous cell carcinoma, two in patients with adenocarcinoma, and 5 included patients with both cell types. All utilized platinum/FU chemotherapy with $20-50$ Gy of radiation delivered concurrently. As in the preoperative chemotherapy trials, the majority of these trials were underpowered to satisfactorily answer the question. The largest of these trials were reported by Burmeister, Bosset, and Van Hagen, but only the latter trial (CROSS) met its primary endpoint of improved overall survival (7). CROSS was a multi-institutional phase III trial that randomized 366 patients with either squamous cell or adenocarcinoma of the esophagus/GEJ to preoperative chemoradiation followed by surgery (CRS) or surgery alone (S) (Table 2). Nearly $23 \%$ of patients had squamous cell cancer. Preoperative chemoradiotherapy consisted of weekly carboplatin and paclitaxel for 5 weeks with concurrent radiotherapy of 41.4 Gy delivered in 23 fractions. Ninetyfive percent of patients in the CRS arm completed their assigned preoperative treatment. The $\mathrm{R} 0$ resection rate was significantly higher in the CRS group versus surgery alone (92\% vs. 69\%). The pathologic complete response was $29 \%$ (23\% for adenocarcinoma versus $49 \%$ for squamous cell carcinoma). Five-year survival was significantly higher in the CRS arm (47\% vs. $33 \%)$. The difference in overall survival was far more prominent in patients with squamous cell carcinoma (median survival: CRS: 81 months, S: 21 months) than adenocarcinoma (CRS: 43 months, S: 27 months), likely reflecting the higher pathological response rate in patients with squamous cell cancers. Patients in the CRS arm had lower rates of locoregional (22\% vs. 38\%) and distant failures (39\% vs. 48\%).

The CROSS trial established the superiority of preoperative chemoradiation to surgery alone in patients with locally advanced esophageal cancer and confirmed CRS as a standard of care for squamous cell and adenocarcinoma of the esophagus and GEJ. However, there are potentially important limitations to the CROSS trial, including its wider applicability to patients with esophageal cancer. For example, fewer than $45 \%$ of the 837 patients screened for this trial were eventually eligible for randomization. 


\begin{tabular}{|c|c|c|c|c|c|c|c|c|c|c|}
\hline Reference & \multicolumn{2}{|c|}{$\begin{array}{l}\text { Pathological complete } \\
\text { response (\%) }\end{array}$} & \multicolumn{4}{|c|}{ 3-yr survival (\%) } & \multicolumn{2}{|c|}{ 3-yr survival (\%) } & \multicolumn{2}{|c|}{ R0 resection (\%) } \\
\hline $\begin{array}{l}\text { Deng HY, et al. } \\
\text { (2016) (14) }\end{array}$ & 22.1 & 3.7 & 46.3 & 56.8 & 41.0 & 42.8 & 52 & 42 & 89.1 & 76.9 \\
\hline
\end{tabular}

Meta-analysis: pathological complete response rate is significantly improved with neoadjuvant chemoradiotherapy compared to chemotherapy alone. [RR: 5.71; 95\% Cl, (3.06-10.65); $\mathrm{P}<0.001]$; 3-yr survival significantly improved in patient with SCC undergoing neoadjuvant chemoradiotherapy compared to chemotherapy alone. [RR: $1.31 ; 95 \% \mathrm{Cl}$, (1.10-1.58); $\mathrm{P}=0.003$ ]; 3-yr survival not significantly improved in patients with ADC undergoing neoadjuvant chemoradiotherapy compared to chemotherapy alone. [RR: 1.13; 95\% Cl, (0.88-1.45); $\mathrm{P}=0.34$ ]; 3-yr survival significantly improved in patients undergoing neoadjuvant chemotherapy (all cell types) compared to chemotherapy alone. [RR: $1.23 ; 95 \% \mathrm{Cl},(1.06-1.43) ; \mathrm{P}=0.006$ ]; R0 resection rate significantly improved with neoadjuvant chemoradiotherapy compared to neoadjuvant chemotherapy alone. [RR:1.15; 95\% Cl, $(1.08-1.23)$; $\mathrm{P}<0.001$ ]. Accumulating evidence suggests that esophageal SCC responds better to CRS, whereas esophageal ADC responds best to neoadjuvant chemotherapy alone to avoid adverse effects of radiation.

Another factor to consider is that the adjusted hazard ratio for patients with adenocarcinoma was not statistically significant ( $\mathrm{P}$ value: 0.059). These considerations will probably generate more interest in defining the optimal induction strategy for patients with adenocarcinoma of the esophagus.

\section{What is the optimal preoperative strategy?}

Meta-analysis has been performed to determine the best preoperative strategy $(14,15)$. A recent meta-analysis (14) identified 5 randomized trials that compared preoperative chemotherapy with preoperative chemoradiation (Table 3). Two trials were done exclusively in patients with adenocarcinoma, two in patients with squamous cell carcinoma and one trial that included both cell types. The chemotherapy regimen in all trials was Cis/FU and the radiotherapy dose varied between 30 and $40 \mathrm{~Gy}$. Notably, all of these trials were either not adequately powered or were closed prematurely and therefore were inadequately powered to detect a survival advantage. The meta-analysis showed that preoperative chemoradiation significantly increased the rates of R0 resection $(89 \% v s$. $77 \%)$ and complete pathological response (22\% vs. $3.7 \%$ ). Nonetheless, a survival advantage was only observed in patients with squamous cell carcinoma (57\% vs. $42.8 \%$ ). In patients with adenocarcinoma, 3-year survival did not differ significantly between preoperative chemoradiation and preoperative chemotherapy ( $46 \%$ vs. $41 \%$ ). As previously stated, since these trials were grossly underpowered, the level of evidence provided is insufficient to make a robust recommendation in favor of a specific induction strategy.

\section{Does esophagectomy have a role after chemoradiation?}

The high pathological complete response rate reported after chemoradiotherapy has prompted some to question the role of esophagectomy after such therapy. This view has been gaining traction in the United States, especially in patients with squamous cell carcinoma. The evidence supporting omission of esophagectomy after chemoradiation is derived mainly from FFCD $9102(10,11)$, a French randomized trial, in which 259 patients with locally advanced esophageal cancer (cT3N0-1M0) were randomly allocated to either chemoradiation followed by surgery or definitive chemoradiation. Nearly $90 \%$ of patients had squamous cell carcinoma. Chemotherapy consisted of two cycles of $\mathrm{FU}$ and $\mathrm{Cis}$ and either conventional (46 Gy in 4.5 weeks) or "split-course" (15 Gy, days 1 to 5 and 22 to 26) concurrent radiotherapy. Patients who responded were then randomized to either surgery or continuation of chemoradiotherapy (three cycles of FU and $\mathrm{Cis}$ and either conventional 20 Gy or split-course $15 \mathrm{~Gy}$ radiotherapy). Two-year survival and median survival were both higher in the chemoradiotherapy alone arm $(40 \%$ vs. $34 \%$ and 19.3 vs. 17.7 months, respectively). However, two-year local control rate was significantly better in the surgery arm (66\% vs. 57\%) and fewer patients required stent placement after esophagectomy ( $5 \%$ vs. $32 \%)$. Three-month mortality was much higher in the surgery group $(9.3 \%$ vs. $0.8 \%)$. The authors thus concluded that 
in patients with squamous cell carcinoma who respond to chemoradiotherapy, there is no benefit from the addition of surgery after chemoradiation compared with the continuation of additional chemoradiation. However, the trial has been criticized for only randomizing responders to chemoradiation, since it can be argued that non-responders would be as or even more likely to benefit from surgical resection. Furthermore, the bulk of the available level I evidence on chemoradiation and squamous cell cancer of the esophagus is derived from trials of trimodality therapy versus surgery alone. Therefore, we prefer to treat patients with locally advanced squamous cell cancer with induction chemoradiation with preoperative intent. Esophagectomy may be omitted in patients with poor performance status or those with prohibitive co-morbidities. Patients with a complete clinical response, defined as physiological uptake on PET scanning and absence of endoscopic evidence of disease, may also elect to defer surgical resection. The data supporting non-operative therapy in patients with adenocarcinoma are even less compelling. Pathological complete responses after chemoradiation in this subset of patients ranges between $15 \%$ and $25 \%$. Notably, we and others have shown that even in patients with adenocarcinoma who achieve complete clinical response, as previously defined after chemoradiation, approximately $30-40 \%$ have residual disease $(16,17)$. Therefore, absent compelling contraindications, surgical resection remains an essential component of the treatment plan.

\section{Adjuvant therapy}

Few trials have evaluated postoperative therapy for esophageal cancer (18-20), and much of the data emanates from trials designed using a perioperative regimen, mostly for gastric cancers. An important early trial, the SWOG-directed Intergroup 0116, compared adjuvant chemoradiotherapy following curative resection of gastric cancer versus observation $(18,19)$. Twenty percent of these patients had primary GE junction tumors, and therefore the results have often been used to justify similar adjuvant treatments for esophageal cancer. Following R0 resection of gastric or GE adenocarcinomas, patients were randomly assigned to either observation or chemoradiotherapy. Chemoradiotherapy consisted of bolus $\mathrm{FU}$ and leucovorin (LV) before, during, and after radiotherapy. The total radiation dosage was $45 \mathrm{~Gy}$. Treatment toxicity was significant, and the trial regimen is no longer used. Only $64 \%$ of patients in the adjuvant chemoradiotherapy group completed the full course. Nearly
$70 \%$ of patients had $\mathrm{T} 3$ or T4 tumors, and over $80 \%$ had nodal metastasis. Median overall survival in the surgery alone group was 27 months compared to 36 months in the chemoradiotherapy group. Local recurrence was higher in the surgery alone group compared to the chemoradiotherapy group (29\% vs. 19\%). While the Intergroup trial offers some support to the use of adjuvant therapy, particularly in node-positive patients, the fact that the trial was designed to evaluate advanced gastric cancers, together with the significant toxicity of its regimen, make its relevance to the treatment of esophageal cancer less clear. Furthermore, the majority of patients in the trial did not have what most would consider oncologically adequate lymph node dissection.

At the 2016 American Society of Clinical Oncology annual meeting, the results of a large phase III trial, the CRITICS study, were presented (21). Although this trial was designed to evaluate adjuvant therapy in gastric cancers, $17 \%$ of patients had GE junction adenocarcinomas. Patients with Ib-Iva resectable gastric adenocarcinoma were randomized to either preoperative chemotherapy followed by surgery and adjuvant chemotherapy versus preoperative chemotherapy followed by surgery and adjuvant chemoradiotherapy. Neoadjuvant chemotherapy in both arms consisted of 3 cycles of epirubicin, cisplatin/oxaliplatin, and capecitabine (ECC/ EOC). Surgery was either total or partial gastrectomy with en bloc lymphadenectomy and a minimum of 15 lymph nodes removed. Adjuvant chemotherapy consisted of an additional 3 courses of ECC/EOC and adjuvant chemoradiotherapy consisted of 45 Gy total dose radiation with concurrent weekly Cis and daily capecitabine. The primary endpoint was overall survival. Five-year survival was $41.3 \%$ in the chemotherapy group versus $40.9 \%$ in the chemoradiotherapy group. These results suggest that performing an adequate node dissection might eliminate the need for radiation as an added modality of local control.

The data supporting adjuvant therapy are soft, and therefore we place priority on neoadjuvant treatment. We believe patients better tolerate treatment in this setting, particularly in light of the high rate of treatment drop out in the adjuvant trials as noted above. We reserve adjuvant chemotherapy for those rare, highly selected patientspatients with T1 tumors, for example-who do not receive neoadjuvant treatment and are found to have occult nodal disease at the time of surgery.

\section{Future directions}

There no longer remains any question as to whether 
preoperative treatment should be given in some manner for patients with resectable locoregionally advanced esophageal cancer. However, the best preoperative treatment is still not clearly established. Several ongoing randomized trials are investigating the optimal preoperative regimen. For example, the FLOT-4 trial (22), a phase II/III trial from Germany, has randomly allocated 714 patients with cancer of the stomach and GEJ to the MAGIC regimen (ECF/ECX) or to perioperative fluorouracil, leucovorin, oxaliplatin, and docetaxel (FLOT). Preliminary analysis of the phase II portion of the trial shows that patients receiving the FLOT regimen have a higher rate of pathological complete response and minimal residual disease compared with patients receiving the MAGIC regimen ( $29 \%$ vs. $15 \%)$. Three additional phase III trials are directly comparing preoperative chemotherapy with preoperative chemoradiation. The Neo-AEGIS trial (23) will randomize 366 patients with adenocarcinoma of the GEJ and esophagus to the MAGIC regimen followed by surgery or the CROSS protocol. The ESOPEC trial (24) will randomize 438 patients with adenocarcinoma of the esophagus to either the previously mentioned FLOT regimen followed by surgery or the CROSS protocol. Finally the NExT trial (JCOG 1109), a 3-arm phase II trial (25), will randomize over 500 patients with squamous cell cancer to either of two chemotherapy regimens (Cis/ FU or DCF) followed by surgery or chemoradiation followed by surgery. It is hoped that these trials will provide clarity regarding the optimal induction strategy. Finally, the introduction of immune checkpoint inhibitors has literally transformed the care of patients with melanoma, non-small cell lung cancer, kidney, and bladder cancer. Several phase III trials are currently ongoing using immune checkpoint inhibitors in patients with advanced gastric and esophageal cancers based on promising results from early phase trials. Our group has recently initiated a phase II trial combining the immune checkpoint inhibitor pembrolizumab (antiPD1) with preoperative chemoradiation followed by esophagectomy and maintenance immunotherapy in patients with esophageal adenocarcinoma. The promise that immunotherapy will prove as transformative for esophageal cancer patients as it has been for patients with other solid tumors is tantalizing indeed.

\section{Concluding remarks}

Our group was an early advocate of neoadjuvant chemotherapy alone followed by surgical resection, and we did not routinely use radiation therapy in the preoperative setting. Given that most patients who succumb to esophageal cancer do so because of distant disease, we believe that chemotherapy is an important component of treatment, and we have typically administered such treatment in the neoadjuvant setting. However, we also believe that by performing a more radical en bloc resection, we can achieve higher rates of $\mathrm{R} 0$ resection and superior local control, which is in our view the primary benefit of radiation therapy, yet without subjecting patients to the added potential toxicity and post-operative adverse events that may result from trimodality therapy (26-29). Spicer recently reported (30) on the experience of three large institutions, including our own, with en bloc resection for patients with cT3N1 esophageal cancer treated with either preoperative chemoradiation (100 patients) or preoperative chemotherapy (114 patients). He found no difference between the two groups of patients in either overall or disease-free survival. Notably, there was no difference between the groups in locoregional recurrence rates suggesting that an en bloc esophagectomy after chemotherapy alone achieves local control rates comparable to those reported after trimodality therapy, thus emphasizing the role of surgery as a sufficient modality for local control. A more streamlined bimodality approach serves a suitable platform for the addition of novel additional therapies such as targeted or immunotherapy.

\section{Acknowledgements}

None.

\section{Footnote}

Conflicts of Interest: The authors have no conflicts of interest to declare.

\section{References}

1. Kelsen DP, Winter KA, Gunderson LL, et al. Longterm results of RTOG trial 8911 (USA Intergroup 113): a random assignment trial comparison of chemotherapy followed by surgery compared with surgery alone for esophageal cancer. J Clin Oncol;25:3719-25.

2. Allum WH, Stenning SP, Bancewicz J, et al. Long-term results of a randomized trial of surgery with or without preoperative chemotherapy in esophageal cancer. J Clin Oncol 2009;27:5062-7. 
3. Cunningham D, Allum WH, Stenning SP, et al. Perioperative chemotherapy versus surgery alone for resectable gastroesophageal cancer. $\mathrm{N}$ Engl J Med 2006;355:11-20.

4. Ychou M, Boige V, Pignon JP, et al. Perioperative chemotherapy compared with surgery alone for resectable gastroesophageal adenocarcinoma: a FNCLCC and FFCD multicenter phase III trial. J Clin Oncol 2011;29:1715-21.

5. Kelsen DP, Ginsberg R, Pajak TF, et al. Chemotherapy followed by surgery compared with surgery alone for localized esophageal cancer. N Engl J Med 1998;339:1979-84.

6. Law S, Fok M, Chow S, et al. Preoperative chemotherapy versus surgical therapy alone for squamous cell carcinoma of the esophagus: a prospective randomized trial. J Thorac Cardiovasc Surg 1997;114:210-7.

7. van Hagen P, Hulshof MC, van Lanschot JJ, et al. Preoperative chemoradiotherapy for esophageal or junctional cancer. N Engl J Med 2012;366:2074-84.

8. Bedenne L, Michel P, Bouché O, et al. Chemoradiation followed by surgery compared with chemoradiation alone in squamous cancer of the esophagus: FFCD 9102. J Clin Oncol 2007;25:1160-8.

9. Vincent J, Mareitte C, Pezet D, et al. Early surgery for failure after chemoradiation in operable thoracic oesophageal cancer. Analysis of the non-randomised patients in FFCD 9102 phase III trial: Chemoradiation followed by surgery versus chemoradiation alone. Eur J Cancer 2015;51:1683-93.

10. Urba SG, Orringer MB, Turrisi A, et al. Randomized trial of preoperative chemoradiation versus surgery alone in patients with locoregional esophageal carcinoma. J Clin Oncol 2001;19:305-13.

11. Mariette C, Dahan L, Mornex F, et al. Surgery alone versus chemoradiotherapy followed by surgery for stage I and II esophageal cancer: final analysis of randomized controlled phase III trial FFCD 9901. J Clin Oncol 2014;32:2416-22.

12. Burmeister BH, Thomas JM, Burmeister EA, et al. Is concurrent radiation therapy required in patients receiving preoperative chemotherapy for adenocarcinoma of the oesophagus? A randomized phase II trial. Eur J Cancer 2011;47:354-60.

13. Yang H, Fu J, Liu M, et al. A phase III clinical trial of neoaduvant chemoradiotherapy followed by surgery versus surgery alone for localy advanced squamous cell carcinoma of the esophagus. Ann Oncol 2014;32:abstr TPS4146.

14. Deng HY, Wang WP, Wang YC, et al. Neoadjuvant chemoradiotherapy or chemotherapy? A comprehensive systematic review and meta-analysis of the options for neoadjuvant therapy for treating oesophageal cancer. Eur J Cardiothorac Surg 2016. [Epub ahead of print].

15. Sjoquist KM, Burmeister BH, Smithers BM, et al. Survival after neoadjuvant chemotherapy or chemoradiotherapy for resectable oesophageal carcinoma: an updated metaanalysis. Lancet Oncol 2011;12:681-92.

16. Stiles BM, Salzler G, Jorgensen A, et al. Complete metabolic response is not uniformly predictive of complete pathologic response after induction therapy for esophageal cancer. Ann Thorac Surg 2013;96:1820-5.

17. Cheedella NK, Suzuki A, Xiao L, et al. Association between clinical complete response and pathological complete response after preoperative chemoradiation in patients with gastroesophageal cancer: analysis in a large cohort. Ann Oncol 2013;24:1262-6.

18. Macdonald JS, Smalley ST, Benedetti J, et al. Chemoradiotherapy after surgery compared with surgery alone for adenocarcinoma of the stomach of gastroesophageal junction. N Engl J Med 2001;345:725-30.

19. Smalley SR, Benedetti JK, Haller DG, et al. Updated analysis of SWOG-directed Intergroup Study 0116: a phase III trial of adjuvant radiochemotherapy versus observation after curative gastric cancer resection. J Clin Oncol 2012;30:2327-33.

20. Almhanna K, Shridhar R, Meredith KL. Neoadjuvant or adjuvant therapy for resectable esophageal cancer: is there a standard of care? Cancer Control 2013;20:89-96.

21. Verheij M, Jansen EP, Cats AC, et al. A multicenter randomized phase III trial of neo-adjuvant chemotherapy followed by surgery and chemotherapy or by surgery and chemoradiotherapy in resectable gastric cancer: First results from the CRITICS study. J Clin Oncol 2016;34:abstr 4000.

22. Schulz C, Kullmann F, Kunzmann V, et al. NeoFLOT: Multicenter phase II study of perioperative chemotherapy in resectable adenocarcinomna of the gastroesophageal junction or gastric adenocarcinoma - Very good response predominantly in patients with intestinal type tumors. Int J Cancer 2015;137:678-85.

23. Keegan N, Keane F, Cuffe S, et al. Neo-AEGIS: A randomized clinical trial of neoadjuvant and adjuvant chemotherapy (modified MAGIC regimen) versus chemoradiation (CROSS protocol) in adenocarcinoma of the esophagus and esophagogastric junction. J Clin Oncol 2014;32:abstr TPS4145.

24. Hoeppner J, Lordick F, Brunner T, et al. ESOPEC: 
prospective randomized controlled multicenter phase III trial comparing perioperative chemotherapy (FLOT protocol) to neoadjuvant chemoradiation (CROSS protocol) in patients with adenocarcinoma of the esophagus (NCT02509286). BMC Cancer 2016;16:503.

25. Kato K, Igaki H, Ito Y, et al. Next study (JCOG 1109): A three-arm randomized phase III study comparing preoperative $\mathrm{CDDP}+5-\mathrm{FU}(\mathrm{CF})$ versus docetaxel + CF-radiation followed by esophagectomy with D2-3 lymphadenectomy for locally advanced esophageal squamous cell cancer. J Clin Oncol 2013;31:abstr TPS4152.

26. Altorki NK. The rationale for radical resection. Surg Oncol Clin N Am. 1999;8:295-305.

27. Altorki N, Kent M, Ferrara C, et al. Three-field lymph

Cite this article as: Altorki N, Harrison S. What is the role of neoadjuvant chemotherapy, radiation, and adjuvant treatment in resectable esophageal cancer? Ann Cardiothorac Surg 2017;6(2):167-174. doi: 10.21037/acs.2017.03.16 node dissection for squamous cell and adenocarcinoma of the esophagus. Ann Surg 2002;236:177-83.

28. Lee PC, Farooq MM, Port JL, et al. Predictors of recurrence and disease-free survival in patients with completely resected esophageal carcinoma. J Thorac Cardiovasc Surg 2011;141:1196-206.

29. Stiles, BM, Christos P, Port JL, et al. Predictors of survival in patients with persistent nodal metastases after preoperative chemotherapy for esophageal cancer. J Thorac Cardiovasc Surg 2010;139:387-94.

30. Spicer JD, Stiles BM, Sudarshan M, et al. Preoperative chemoradiation therapy versus chemotherapy in patients undergoing modified en bloc esophagectomy for locally advanced esophageal adenocarcinoma: Is radiotherapy beneficial? Ann Thorac Surg 2016;101:1262-9. 